\title{
MANUAL DE EXERCÍCIOS DOMICILIARES PARA A PESSOA COM TRAUMATISMO CRÂNIOENCEFÁLICO
}

\section{ARTIGO ORIGINAL}

BORGES, Carolina dos Santos Silva ${ }^{1}$

SILVA, Daiane Medeiros da ${ }^{2}$

TOMAZ, Renata Ramos ${ }^{3}$

CERQUEIRA, Ana Carolina Dantas Rocha ${ }^{4}$

\footnotetext{
${ }^{1}$ Mestrado em Saúde da Família pela Faculdade de Enfermagem Nova Esperança João Pessoa. Pós-graduação em Coluna Vertebral e em Disfunções Biomecânicas e Posturais pela Faculdade Einstein - Salvador. Graduada em Fisioterapia pelo Centro Universitário Maurício de Nassau - João Pessoa.
}

2 Doutorado em Enfermagem pela Universidade Federal da Paraíba - UFPB. Mestrado em Enfermagem pela Universidade Federal da Paraíba - UFPB. Especialização em Enfermagem do Trabalho. Consultoria Brasileira de Ensino, Pesquisa e Extensão, CBPEX. Graduação em Licenciatura em Enfermagem pela Universidade Federal da Paraíba - UFPB. Graduação em enfermagem pela Universidade Federal da Paraíba - UFPB.

${ }^{3}$ Doutorado em Fisioterapia pela Universidade Federal do Rio Grande do Norte, UFRN. Mestrado em Fisioterapia pela Universidade Federal do Rio Grande do Norte, UFRN. Especialização em Especialista Profissional em Fisioterapia Respiratória pela Associação Brasileira de Fisioterapia Cardiorrespiratória e Fisioterapia e Terapia Intensiva- ASSOBRAFIR. Graduação em Fisioterapia pelo Centro Universitário de João Pessoa- UNIPÊ.

${ }^{4}$ Doutorado em Enfermagem pela Universidade Federal do Ceará- UFC. Mestrado em Saúde Pública pela Universidade Estadual da Paraíba-UEPB. Especialização em andamento em Especialização em Saúde Mental e Rede de Atenção Psicossocial pela 
LUCENA, Larissa Coutinho de ${ }^{5}$

RODRIGUES NETO, Gabriel ${ }^{6}$

BORGES, Carolina dos Santos Silva. Et al. Manual de Exercícios Domiciliares para a Pessoa com Traumatismo Crânioencefálico. Revista Científica Multidisciplinar Núcleo do Conhecimento. Ano 05, Ed. 10, Vol. 22, pp. 101-119. Outubro de 2020. ISSN: 2448-0959, Link de acesso: https://www.nucleodoconhecimento.com.br/saude/exercicios-domiciliares

\section{RESUMO}

O Traumatismo Cranioencefálico (TCE) é uma lesão traumática que compromete as estruturas e a função do crânio. No Brasil, este evento atinge, anualmente, 500 mil pessoas, e cerca de 75 a 100 mil vão a óbito, contribuindo para o aumento dos gastos diretos e indiretos no Sistema Único de Saúde (SUS), assim como, no setor previdenciário. Muitas pessoas com deficiências enfrentam obstáculos no acesso aos serviços de reabilitação, e, considerando as sequelas crônicas desta condição em saúde, objetivou-se construir uma tecnologia educativa que promovesse a ininterrupção da reabilitação no domicílio a este público exercida pela Atenção Básica (AB) e a participação do cuidador nesse processo. Trata-se de um estudo

Instituto de Ensino Superior Múltiplo. Graduação em Enfermagem pela Universidade Estadual da Paraíba- UEPB.

${ }^{5}$ Doutorado em Fisioterapia pela Universidade Federal do Rio Grande do Norte UFRN. Mestrado em Fisioterapia pela Universidade Federal do Rio Grande do Norte, UFRN. Especialização em Fisioterapia Neurofuncional pela Faculdade Estácio do Recife, Estácio FIR. Graduação em Fisioterapia pela Universidade Federal da Paraíba, UFPB.

${ }^{6}$ Orientador. Doutorado em Educação Física pela Universidade Federal da Paraíba, UFPB. Mestrado em Educação Física pela Universidade Federal do Rio de Janeiro, UFRJ. Especialização em Fisiologia do Exercício pela Faculdade Integrada de Patos - FIP. Graduação em Educação Física pela Universidade Federal da Paraíba, UFPB. 
metodológico que, por meio de uma revisão da literatura, construiu um manual educativo. $\mathrm{O}$ construto é ilustrativo e contém exercícios reabilitadores direcionados para as três fases do TCE, e estes são divididos em módulos e diferenciados por cores, podendo ser utilizados tanto por crianças como adultos. O material educativo caracteriza-se por ser um recurso terapêutico de baixo custo e tem como objetivo promover a educação dos cuidadores de TCE e a qualidade de vida da pessoa com tal lesão, por meio da educação em saúde e reabilitação exercida pelo fisioterapeuta das equipes de saúde no âmbito domiciliar.

Palavras-chave: Lesões encefálicas traumáticas, saúde da família, reabilitação, terapia por exercício, cuidadores.

\section{INTRODUÇÃO}

O Traumatismo Cranioencefálico (TCE) é uma lesão de origem traumática que gera comprometimentos estruturais e funcionais no encéfalo, tal como desencadeia a ocorrência de sequelas temporárias ou permanentes de ordem cognitiva e sensóriomotoras (FUENTES et al., 2014; MORAES et al., 2014; NITRINI; BACHESCHI, 2015). No Brasil, estima-se que, a cada ano, 500 mil pessoas são acometidas pelo TCE, e cerca de 75 a 100 mil pessoas vão a óbito (ASSOCIAÇÃO DE MEDICINA INTENSIVA BRASILEIRA, 2014).

Fukujima (2013) relata que, apenas no ano 2012, ocorreram 998.994 internações por TCE, e o valor gasto foi de $R \$ 1$ bilhão de reais pelo Sistema Único de Saúde (SUS), enquanto o Centro de Pesquisa e Economia do Seguro (2016) revelou que, em 2014, o país gastou em torno de $R \$ 118$ bilhões de reais do PIB em indenizações por invalidez do seguro de Danos Pessoais Causados por Veículos Automotores de via Terrestre (DPVAT). É importante frisar também que, devido à permanência dos inúmeros comprometimentos decorrentes do trauma encefálico, os gastos não afetam apenas os serviços de saúde pública, mas também gera custos ao setor previdenciário. 
Atualmente, a pessoa com TCE é assistida no âmbito do SUS por meio da rede de cuidados à pessoa com deficiência (RCPD). O SUS disponibiliza componentes da rede que objetivam gerar ações de prevenção, promoção da saúde, como também de recuperação e de reabilitação da saúde. Desta forma, são componentes da RCPD: a Atenção Básica $(A B)$ juntamente com o Núcleo ampliado de Saúde da Família e Atenção Básica (NASF - AB) e o Serviço de Atenção Domiciliar (SAD), Atenção Especializada em Reabilitação (AER) e Atenção Hospitalar e de Urgência e Emergência (AHUE) (BRASIL, 2010; BRASIL, 2012; BRASIL, 2017; BRASIL, 2018).

No contexto dos serviços de reabilitação, a $A B$ fornece ações de prevenção, promoção e recuperação da saúde por meio do apoio matricial do NASF - AB. O NASF-AB realiza o cuidado individual e coletivo mediante práticas terapêuticas, educativas e de acolhimento. As ações terapêuticas caracterizam-se por utilizar recursos de baixa densidade tecnológica; as de acolhimento realizam a escuta qualificada, e as ações educativas são executadas por meio de orientações de cuidado que promovem a qualidade de vida do indivíduo (BRASIL, 2010; BRASIL, 2017; BRASIL, 2018).

Vale ressaltar que as limitações funcionais impedem o acesso igualitário aos serviços de saúde. Sendo assim, o NASF- AB e o SAD são responsáveis por promover a assistência domiciliar a pacientes que possuam dificuldades de locomoção e que estejam restritos ao leito (BRASIL, 2012).

Ainda que se disponha de uma rede bem estruturada que oferte, em seus diversos pontos de atenção, ações de reabilitação, há relatos na literatura de que as Pessoas com Deficiências (PCDs) enfrentam obstáculos no acesso aos serviços de reabilitação pública. Os problemas evidenciados são: as limitações funcionais e financeiras para se deslocar ao serviço mais próximo, as filas de espera, dificuldades nos encaminhamentos e a ausência de profissionais de fisioterapia para atender à demanda do território (SOUZA; ROCHA, 2010; CASTRO et al., 2011; SOUZA et al., 2013).

Com base nas dificuldades apontadas na literatura no setor de reabilitação do Brasil e pelas lacunas do conhecimento referente à criação de tecnologias voltadas ao TCE, 
o estudo teve como objetivo construir uma tecnologia educativa direcionada a orientar os cuidadores de TCE e fornecer ações de prevenção das complicações motoras e a promoção da qualidade de vida da pessoa com TCE, de modo que esta promovesse a ininterrupção da reabilitação a este público no âmbito domiciliar com foco na $A B$, assim como a participação do cuidador no processo terapêutico.

\section{MATERIAIS E MÉTODOS}

Trata-se de um estudo de cunho metodológico que envolve a elaboração de uma tecnologia educativa direcionada aos cuidadores de indivíduo com TCE. Para a construção do material, fez-se necessária a divisão em três fases, representadas na Figura 01.

Figura 01: Fases de construção da tecnologia educativa.
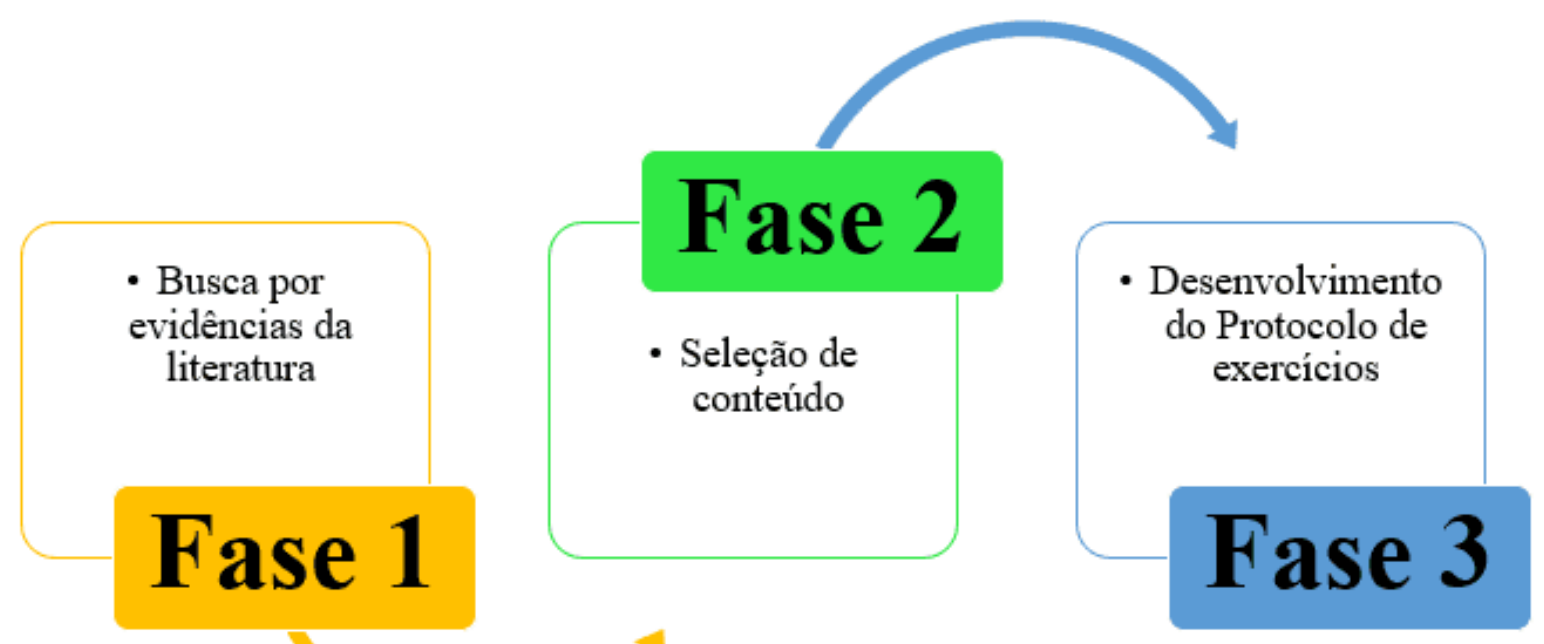

Fonte: BORGES, C. S. S. dos. Dados da Pesquisa (2019).

\section{Fase 1- Levantamento de evidências da literatura}

Nesta fase, buscaram-se relatos na literatura de problemas evidenciados pelos cuidadores e indivíduos com TCE, bem como da existência de protocolos de exercícios terapêuticos direcionados a este público, visando definir os conteúdos relevantes a serem abordados no manual educativo. O levantamento bibliográfico 
ocorreu mediante uma revisão sistemática disponível no estudo "Therapeutic Exercise Protocols in Patients with Traumatic Brain Injury: A Systematic Review", publicado na revista: Journal of Exercise Physiology online, em abril de 2020, no volume 23, número 2.

\section{Fase 2- Seleção do conteúdo}

Nesta fase, iniciou-se a síntese e o agrupamento das informações pertinentes encontradas na literatura científica. Desta forma, foi possível observar quais exercícios seriam mais adequados para o contexto domiciliar, bem como o quanto estes impactariam nas sequelas motoras do indivíduo com TCE. A partir destes, foram selecionados os exercícios e os objetivos específicos para compor o manual educativo (Quadro 1).

\section{Fase 3 - Elaboração do Protocolo de Exercícios Terapêuticos Domiciliares}

Após a seleção do conteúdo, na fase 3 , priorizou-se adaptar os exercícios encontrados na literatura para o âmbito domiciliar. Assim, elaborou-se o protocolo dos exercícios terapêuticos, por meio do registro de fotografias, utilizando objetos simples, de baixo custo e acessíveis no domicílio.

Por conseguinte, criaram-se as instruções dos exercícios de forma clara, objetiva e compreensível a toda a população. Esta etapa tomou como base os conceitos de Escher (2005) acerca da adequação da linguagem dos materiais educativos na área da saúde. Além disso, vale ressaltar que a construção do protocolo está de acordo com os apontamentos da diretriz de prescrição de exercícios descrita por Voight, Hoogenboom e Prentice (2014), que reforça a importância de evitar exageros na prescrição dos exercícios, como também utilizar exercícios simples, para que a aprendizagem seja facilitada.

Desta forma, o protocolo consta de exercícios que obedecem a uma sequência lógica, então, cada módulo está correlacionado a uma determinada fase do TCE. Sendo assim, a descrição dos exercícios selecionados e os objetivos específicos de cada módulo estão disponíveis no quadro abaixo. 
Quadro 01: Distribuição dos objetivos específicos e exercícios selecionados do protocolo.

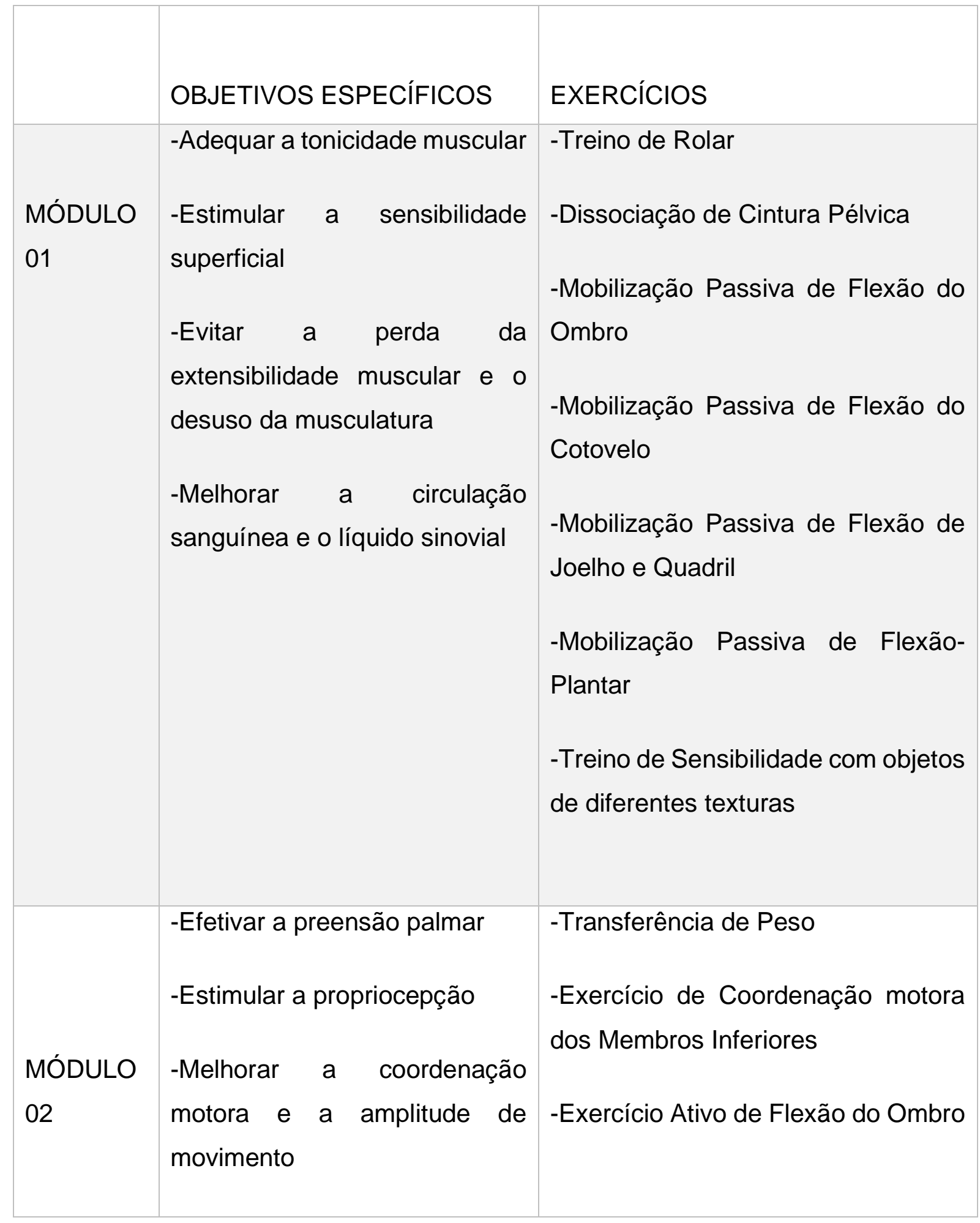




\begin{tabular}{|c|c|c|}
\hline & $\begin{array}{l}\text {-Proporcionar a independência } \\
\text { funcional } \\
\text {-Trabalhar a consciência } \\
\text { corporal }\end{array}$ & $\begin{array}{l}\text {-Exercício Ativo de Flexão e } \\
\text { Dorsiflexão-Plantar } \\
\text {-Treino de Alcance Funcional } \\
\text { utilizando copos } \\
\text {-Treino de Motricidade Fina } \\
\text { utilizando moedas } \\
\text {-Treino de Pinça utilizando bolas de } \\
\text { gude }\end{array}$ \\
\hline MÓL & $\begin{array}{l}\text {-Aperfeiçoar a execução do } \\
\text { movimento; } \\
\text {-Aprimorar a coordenação do } \\
\text { movimento e da motricidade } \\
\text { fina; } \\
\text {-Melhorar a amplitude de } \\
\text { movimento; } \\
\text {-Proporcionar a independência } \\
\text { funcional; } \\
\text {-Trabalhar a lateralidade e a } \\
\text { sincronia do movimento. }\end{array}$ & $\begin{array}{l}\text {-Exercício Ativo de Flexão Anterior } \\
\text { do Ombro } \\
\text {-Exercício Ativo de Abdução e } \\
\text { Adução de Ombro } \\
\text {-Exercício Ativo de Coordenação } \\
\text { motora dos Membros Inferiores com } \\
\text { o cabo de vassoura } \\
\text {-Exercício de Elevação Pélvica } \\
\text { (ponte) } \\
\text {-Exercício Ativo dos Membros } \\
\text { Superiores e Inferiores com } \\
\text { movimentos sincronizados } \\
\text {-Exercício Ativo de Coordenação } \\
\text { motora dos Membros Superiores } \\
\text { com o cabo de vassoura }\end{array}$ \\
\hline
\end{tabular}




\author{
-Exercício de Pinça utilizando grãos \\ de feijão \\ -Exercício de Flexão dos Dedos \\ (caminhada com dedos) \\ -Exercício de Flexão de Dedos com \\ o pegador de roupa.
}

Fonte: BORGES, C. S. S. dos. Dados da Pesquisa (2019).

\title{
RESULTADOS
}

O material educativo construído tem como objetivo amenizar a fragmentação encontrada por pessoas com TCE no acesso aos serviços de reabilitação, como também ser um recurso educativo auxiliador aos fisioterapeutas da $A B$ e aos cuidadores de pessoas com disfunções motoras e sensoriais em decorrência de sequelas de TCE. Desta forma, a sua elaboração com criação de imagens e edição ocorreu no período de 6 de dezembro de 2019 a 10 de janeiro de 2020.

É importante ressaltar que o protocolo inserido no material apresenta três módulos diferenciados por cores, cada uma delas está direcionada a um estágio do TCE, obedecendo às noções da Diretriz de Atenção à Reabilitação da Pessoa com Traumatismo Cranioencefálico (2013). Na primeira parte, destacada na cor verde, priorizaram-se exercícios passivos que são executados pelo fisioterapeuta ou cuidador. A cor verde corresponde à fase aguda do TCE (período do trauma até os sete primeiros dias de evolução).

$\mathrm{Na}$ segunda parte, destacada na cor amarela, estão exercícios ativo-assistivos que compreendem a fase subaguda do TCE (período entre 2 a 16 semanas pós-lesão). Estes exercícios já podem ser executados ativamente pelos indivíduos e auxiliados pelo fisioterapeuta ou cuidadores. Na terceira e última parte, destacada pela cor vermelha, estão exercícios ativos direcionados à fase crônica do TCE (anos após a 
lesão). Os exercícios inseridos no protocolo trabalham: mobilidade, propriocepção, equilíbrio, coordenação e a motricidade fina.

Vale ressaltar que se optou por registrar fotografias de um indivíduo com TCE executando os exercícios propostos no protocolo, almejando a fidedignidade do construto. A partir da finalização das fotografias, iniciou-se a construção do material educativo intitulado "Manual de Exercícios Domiciliares para a Pessoa com Traumatismo Crânioencefálico". A tecnologia consta de uma parte externa composta por: capa e contracapa e a parte interna com: a apresentação, a recomendação, os módulos dos exercícios e a finalização do manual. A imagem da capa e contracapa está disponível na figura 02 e as demais estruturas que compõem o material estão disponíveis na Figura 03.

Figura 02: Capa e contracapa do Manual de Exercícios domiciliares para a Pessoa com Traumatismo Crânioencefálico.

\section{Manual de Exercicios Domiciliares para a pessoa com Traumatismo Cranioencefálico}

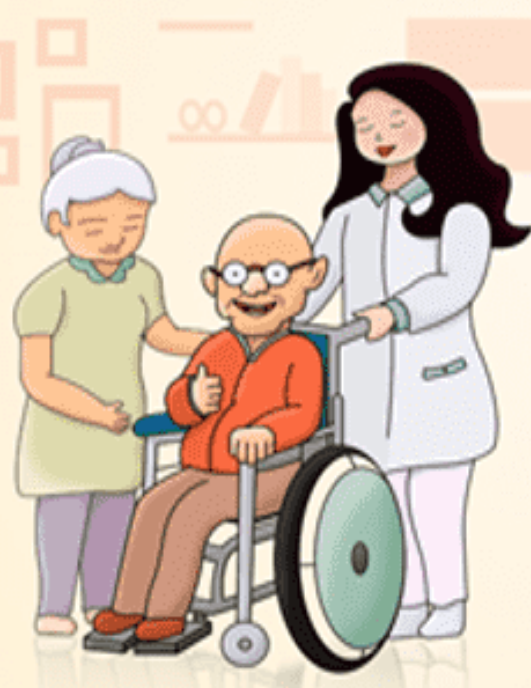

Faculdade de Enfermagem Nova Esperança Programa de Pós-Graduaçăo em Saúde da Familia Mestrado Profissional em Saúde da Familia Criaçảo e idealizaçảo: Carolina dos Santos Silva Borges Orientaçåo: Prof. Dr. Gabriel Rodrigues Neto Revisores: Prof. Dra. Renata Ramos Tomáz Prof. Dra. Daiane Medeiros da Silva

MANUAL DE EXERCICIOS DOMICILIARES PARA A PESSOA COM TRAUMATISMO CRĀNIOENCEFÁLICO

Fonte: BORGES, C. S. S. dos. "Criação da Pesquisadora" (2020). 
A tecnologia consta de uma parte externa composta: por capa e contracapa e a parte interna com: a apresentação, a recomendação, os módulos dos exercícios e a finalização do manual. As demais estruturas que compõem o material estão disponíveis na Figura 03.

Figura 03: Composição do manual de exercícios.

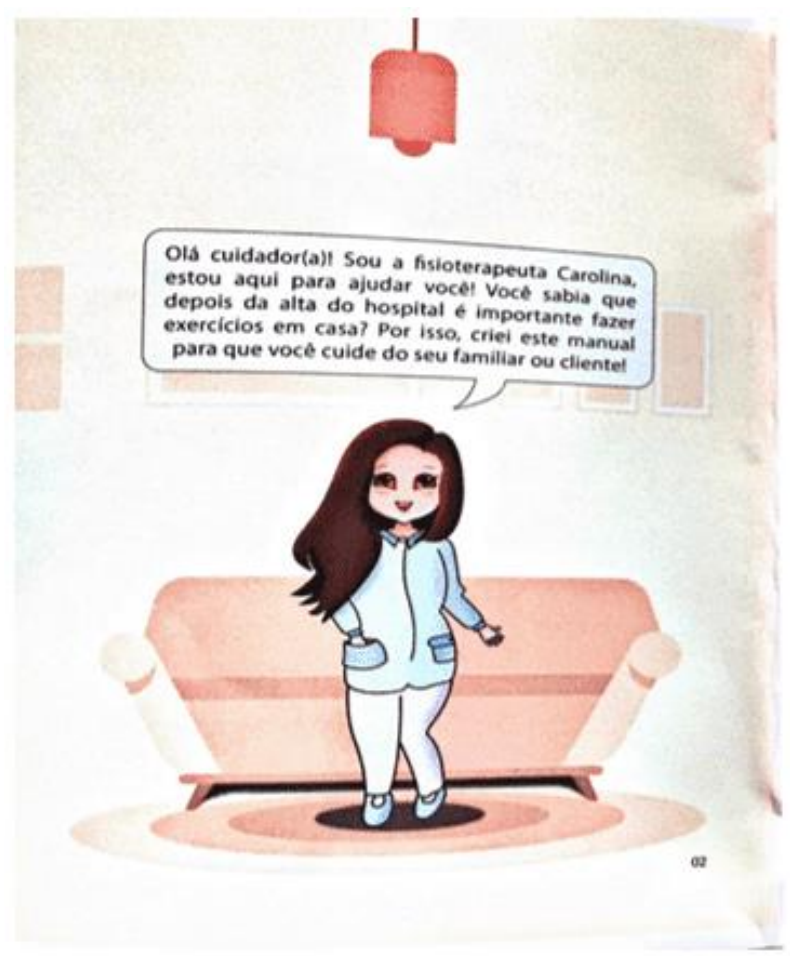

\section{RECOMENDAÇÃO}

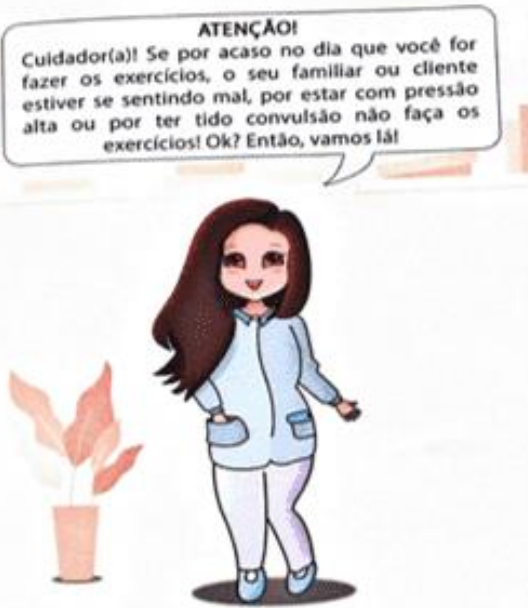




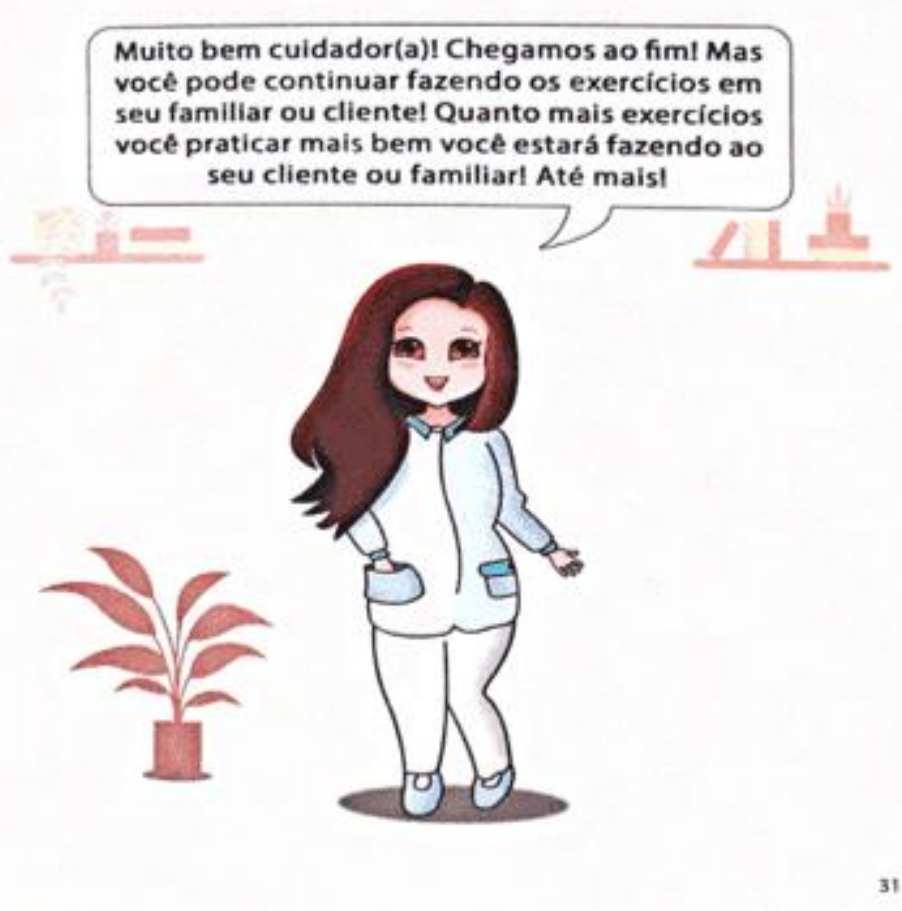

Fonte: BORGES, C. S. S. dos. "Criação da Pesquisadora" (2020).

Durante a construção do manual, objetivou-se criar e utilizar ilustrações que representassem a assistência, o âmbito domiciliar e o vínculo entre o profissional de fisioterapia, o cuidador e a pessoa com TCE. É importante ressaltar que, para aprimorar os aspectos visuais do material, utilizaram-se os apontamentos de Almeida (2017), que reforça a importância de se ter uma boa escolha da cor da fonte em relação ao plano de fundo do material educativo, para que o conteúdo apresentado seja facilmente absorvido e compreendido.

Desta forma, o Software Adobe Photoshop CC foi utilizado para a criação do material desde as ilustrações até a fonte. Sendo assim, a fonte utilizada nos títulos foi a Myriad Pro Regular-70px, e, nas demais informações, utilizou-se Myriad Pro Bold-128px. Além disso, houve também o aprimoramento das fotografias por meio do Software 
Câmera Raw Filter. Nesta etapa, realizou-se o ajustamento de cor, brilho, sombra, balanceamento de cor e de textura das imagens, visando a uma melhor qualidade visual das fotografias. A fase de elaboração textual das instruções fez uso de linguagem coloquial, para que o cuidador, independente do nível de instrução, compreenda facilmente, sem prejuízos.

Portanto, foram utilizados como exemplo de instrução: "Com seu familiar/cliente deitado de barriga para cima, você deve colocar as mãos na pá das costas e levar o paciente até você, ou virar o seu familiar/cliente até ele ficar de frente para você". E, como exemplo de comandos verbais, "Junte as pernas do familiar/cliente, leve as duas pernas do familiar/cliente até a barriga, e depois vire as pernas para o lado onde você está. Depois faça o mesmo do outro lado".

É importante frisar que, como forma de representar o profissional de fisioterapia e apresentar o manual, bem como motivar o cuidador e a pessoa com TCE durante todo o uso do material, criou-se uma personagem "Avatar", denominada "Fisioterapeuta Carolina". A personagem explica cada módulo de forma simples e objetiva. Além disso, atribuíram-se à personagem frases motivadoras, uma vez que, como os estudos de Andelic (2017) e Tornbom, Sunnerhagen e Danielsson (2017) relatam, as pessoas com TCE lidam com a depressão e se sentem desmotivadas em executar exercícios. São exemplos de frases motivadoras da personagem: "Vamos lá, você consegue! Muito bem, você fez um excelente trabalho! O esforço valerá a pena! Você está indo bem!! 
Figura 04: Esboço final "Fisioterapeuta Carolina falando frases motivacionais".

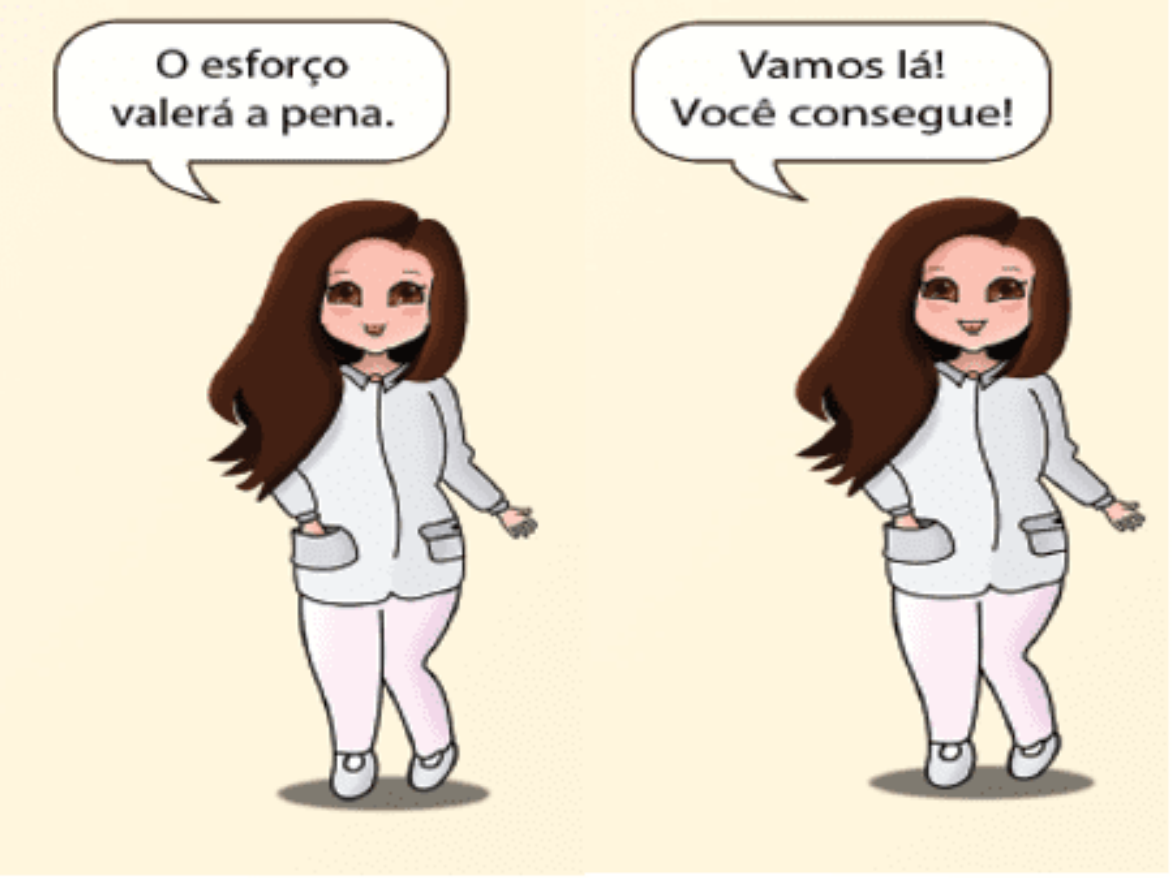

Fonte: BORGES, C. S. S. dos. "Criação da Pesquisadora" (2020).

$\mathrm{Na}$ apresentação dos módulos, como mencionado anteriormente, com diferenciação de cores, em cada módulo, a personagem faz menção de executá-los de forma lenta. São exemplos de frases da personagem: "Cuidador(a), nesta parte, o seu familiar ou cliente já consegue fazer todos os exercícios sozinho(a)! Mas você também pode ajudar ele(a)! Então, explique a ele(a) como se faz! Vamos começar! *Lembrando: sempre faça devagar os exercícios no seu familiar ou cliente, ok? Qualquer dúvida pergunte ao seu fisioterapeuta!" A figura 05 demonstra como os módulos são divididos. 
Figura 05: Apresentação dos módulos.

\section{MÓdULO 1}

Cuidador(a), nesta primeira parte você vai fazer os exercicios no seu familiar ou cliente! Estes exercicios săo importantes para evitar a atrofia.

-Lembrando, sempre faça os exercícios no seu familiar ou cliente devagar $\mathrm{Ok}$ ?

Entāo, vamos começar?

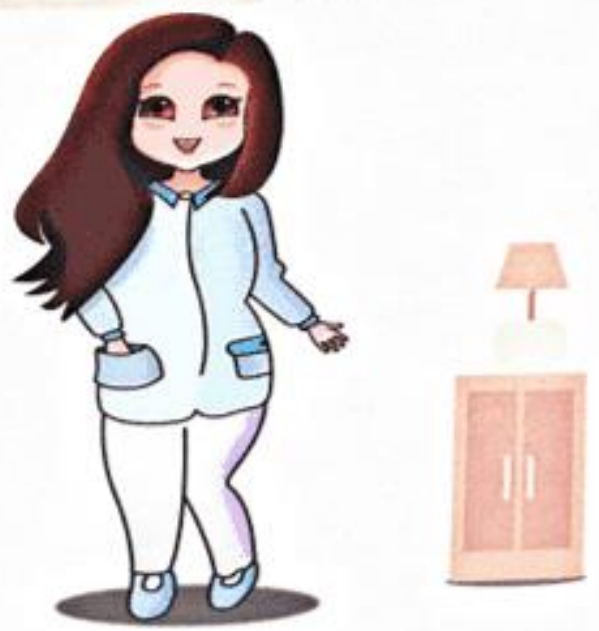




\section{MÓDULO 2}

Cuidador(a), nesta parte o seu familiar ou cliente já consegue fazer alguns exercicios sozinho(a)! Mas vocé também pode ajudar ele(a)! Entåo, explique a ele(a) como se faz! Vamos lá!

*Lembrando, sempre faça os exercicios no seu familiar ou cliente devagar Ok?

Qualquer dúvida pergunte ao seu fisioterapeuta!
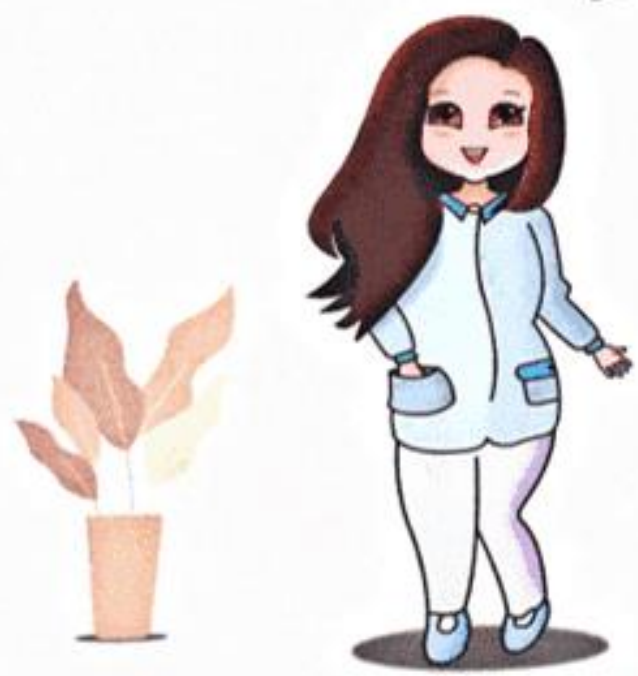


\section{MÓDULO 3}
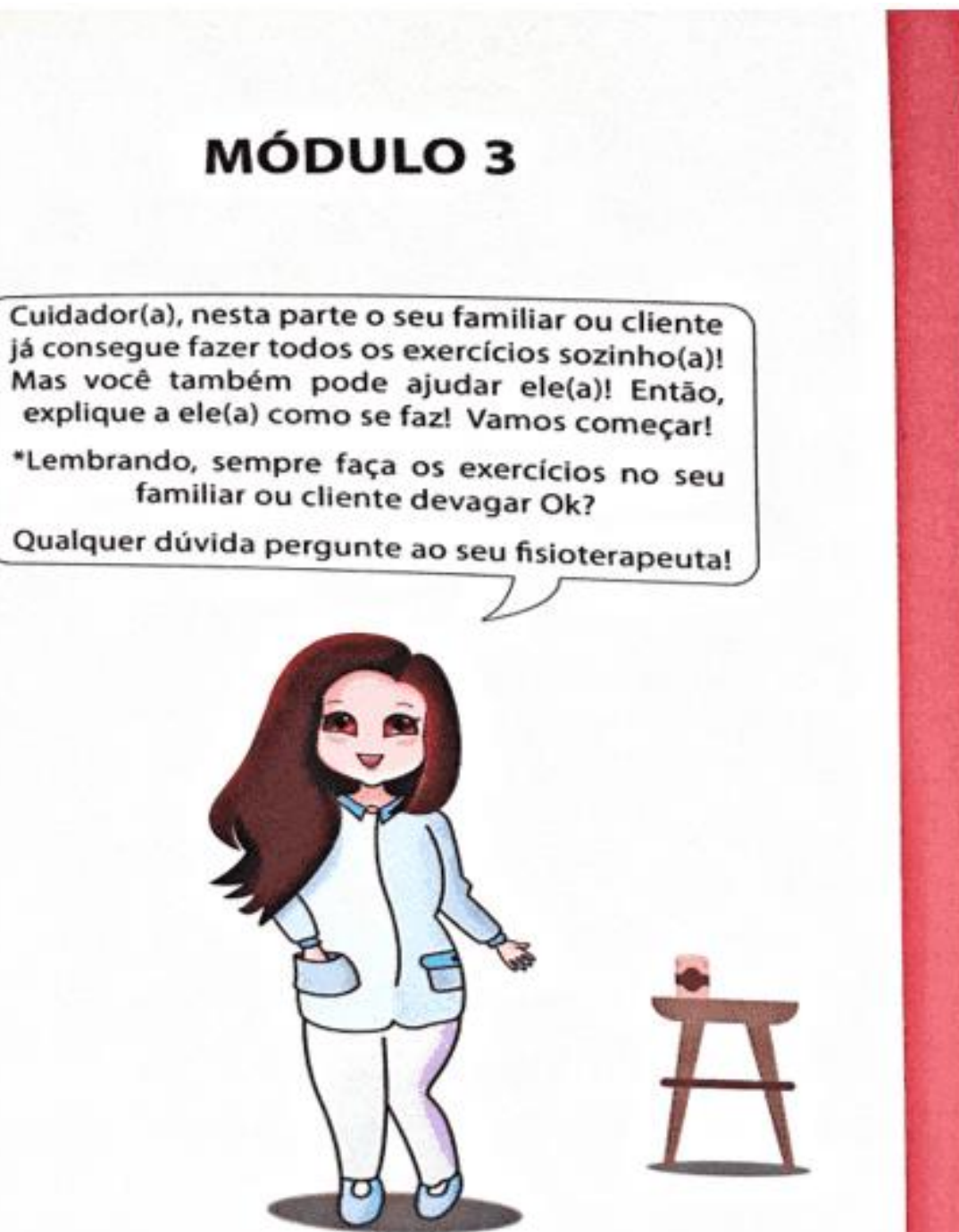

Fonte: BORGES, C. S. S. dos. "Criação da Autora" (2020).

Por fim, a apresentação dos exercícios consiste em instruções e comandos que o cuidador deve realizar ao executar os exercícios. É importante frisar que existem espaços nesta seção para que o fisioterapeuta possa descrever quais serão as repetições e as durações dos exercícios, objetivando a flexibilidade do fisioterapeuta, como também a sua inserção no processo de reabilitação. A forma de apresentação dos exercícios está disponível na Figura 06. 
Figura 06: Forma de apresentação dos exercícios.

\section{EXERCÍCIO 08}

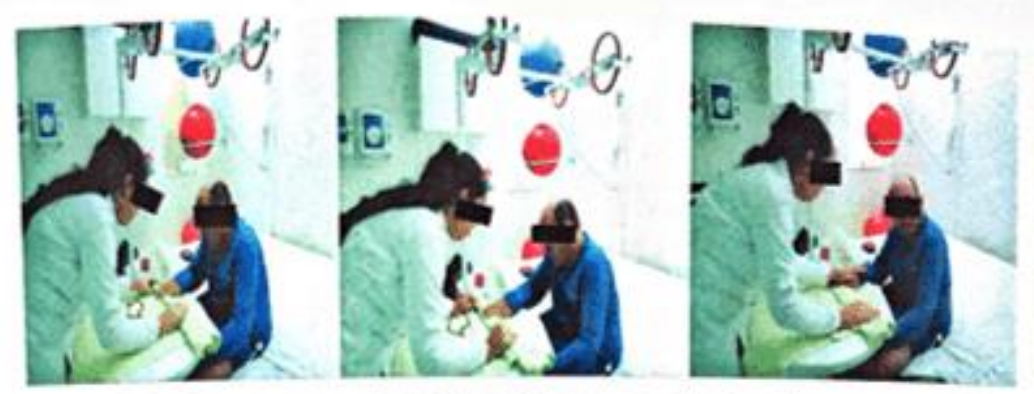

*Instruçáo: Com o seu familiar/cliente sentado á mesa você deve colocar as manos na toalha, e puxar devagar a toalha para que os dedos sejam esticados. "Coloque as manos do seu familiar/cliente em cima da toalha, vá puxando em sua direçáo (para você) até os dedos ficarem "esticados", e depois coloque sua mão em cima, deixando a mão do seu familiar/cliente aberta".

•Repetiçōes:

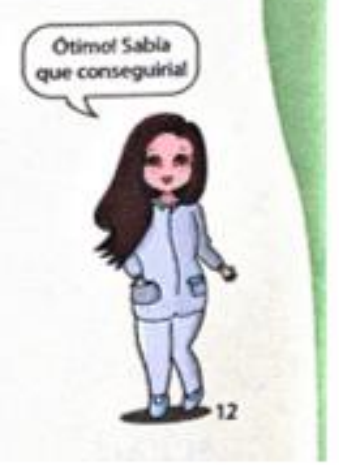

Fonte: BORGES, C. S. S. dos. "Criação da Pesquisadora” (2020).

A versão final do manual é composta por 32 páginas (versão digital em PDF). Entretanto, a versão impressa apresenta 16 páginas frente/verso, uma vez que foi desenvolvida no formato de folha A4 com papel Couché brilhoso, visando a uma melhor adequação do tamanho e da nitidez das imagens. 


\section{DISCUSSÃO}

Nos últimos anos, muitas ferramentas educativas têm sido criadas com o intuito de auxiliar os cuidadores e familiares. É importante ressaltar que a construção de uma tecnologia é fruto da união de conhecimentos, devendo ser produzida de forma coerente (MOREIRA et al., 2018).

Além disso, a elaboração de protocolos de exercícios domiciliares são recursos complementadores da reabilitação motora, uma vez que cabe ao fisioterapeuta a promoção do cuidado e a educação do paciente. Dessa forma, durante as sessões de fisioterapia, é muito comum o fornecimento de materiais ilustrativos e informativos, entretanto, alguns são criados de modo inadequado, o que pode gerar resultados não satisfatórios (AMERICAN PHYSICAL THERAPY, 2005; VOIGHT; HOOGENBOOM; PRENTICE, 2014).

Segundo a Política Nacional de Saúde da Pessoa com Deficiência (2008), pesquisas e ferramentas devem ser criadas, para que facilitem a vida das pessoas que possuem sequelas neurológicas. De acordo com a Organização Mundial da Saúde (2012), a assistência reabilitadora deve também ofertar ações de educação tanto à pessoa com deficiência quanto à família com recursos de baixo custo que promovam o desenvolvimento de habilidades assistenciais e de autocuidado. Sendo assim, a elaboração de recursos e as intervenções de baixo custo que auxiliem esta população possuem extrema relevância.

Diferentemente dos grandes centros de reabilitação que se utilizam de recursos de alta densidade tecnológica, é muito comum a utilização de tecnologias do cuidado leve na $A B$, uma vez que estas favorecem as ações de vincular e acolher o usuário do serviço, a família e as equipes, como também promovem a autonomia por meio das práticas e conhecimentos dos profissionais de saúde (SOUZA, 2013; BRASIL, 2017), visando à adequação ao âmbito da $A B$, reforçando que o material proposto apresenta como característica o fato de ser uma tecnologia do cuidado leve. 
Atualmente, existe apenas um estudo brasileiro da rede SARAH (BRAGA; DA PAZ; YLVISAKER, 2005) e um norte-americano (POWELL et al., 2016) que realizaram intervenções educativas por meio de ferramentas instrutivas em pessoas com TCE. As autoras Braga, da Paz e Ylvisaker (2005) criaram um manual ilustrativo com exercícios domiciliares direcionado aos familiares de crianças com TCE em fase crônica.

Diferentemente, Powell et al. (2016) utilizaram um protocolo de exercícios e orientaram, por meio do telefone, os cuidadores de pacientes com TCE moderado e severo de um Centro de Trauma I, ambos evidenciando que as intervenções educativas proporcionaram resultados satisfatórios no que concerne à melhora do apoio aos cuidadores de sobreviventes de trauma encefálico.

É importante frisar que estes estudos não disponibilizaram na íntegra o construto ou protocolo para que outras pessoas tenham acesso, como também não são direcionados para o âmbito da $\mathrm{AB}$. Vale ressaltar que a ferramenta construída neste estudo consta de exercícios que englobam as fases: aguda, subaguda e crônica do TCE, podendo ser executados tanto por adultos como crianças. Além disso, o protocolo criado permite que os exercícios sejam realizados com utensílios facilmente encontrados no domicílio.

Ademais, existem, na literatura, estudos que criaram manuais educativos para outras patologias. De acordo com Amaral et al. (2005), os materiais são capazes de instruir os pacientes na realização do protocolo sem a supervisão do profissional. Corroborando Assis et al. (2015), enfatiza-se que a entrega do material ao cuidador ou ao paciente é efetivo e que não é necessária a supervisão presencial do fisioterapeuta. Para Petito et al. (2012) e Matos et al. (2013), os manuais são eficientes na recuperação funcional, uma vez que são recursos acessíveis e promovem a união dos profissionais de saúde com a família.

Segundo Silva et al. (2014), os manuais promovem a educação permanente e servem como um meio de consulta durante a reabilitação e depois dela. Além disso, Souza e Knobel (2019) ressaltam que é comum os profissionais de saúde fornecerem muitas 
informações aos cuidadores e familiares, porém muitos não recordam, e a entrega de manuais instrutivos é uma forma de recordação das orientações.

Os estudos de Thinen e Moraes (2013) e Guedes e Silva (2019) confirmam que, após a entrega dos manuais, foi possível observar a melhora na execução das ações dos cuidadores, sendo fatores contribuintes para o preparo do cuidador e a minimização das interrupções da reabilitação.

Assim, o construto tem como base as diretrizes da Política Nacional de Saúde da Pessoa com Deficiência (2019) e caracteriza-se por ser um ferramenta educativa básica e de baixo custo, que busca promover a qualidade de vida direcionada ao âmbito da $A B$, podendo, entretanto, ser utilizado também em qualquer ponto de atenção da RCPD ou no setor privado. Ressalta-se também que a tecnologia é pioneira, já que não se tem relatos na literatura de tecnologias com as mesmas característica da proposta.

Vale ressaltar que o manual educativo está disponível também na versão digital, uma vez que foi criado um link, para que mais pessoas tenham acesso de forma gratuita. Assim, almeja-se que o estudo proposto seja utilizado também como uma ferramenta de divulgação do construto. O link do manual está disponível logo abaixo: https://drive.google.com/file/d/1JzHWr14PAJLs0xyv7ZBjEor-

6miwufD6/view?usp=sharing.

Além disso, a pesquisa teve como principal limitação a escassez de estudos no que concerne a protocolos de exercícios para este público, possibilitando, assim, um maior aprofundamento da temática. É importante destacar que o manual não tem o intuito de substituição do fisioterapeuta, e sim apenas ser uma ferramenta auxiliadora durante as visitas domiciliares, como também um recurso de consulta aos cuidadores ou familiares que estabeleça a continuidade da reabilitação da pessoa com TCE. 


\section{CONCLUSÃO}

Os problemas evidenciados na literatura e a revisão sistemática de protocolos de exercícios possibilitaram, de forma metodológica, a construção de uma tecnologia educativa com o intuito de promover a educação dos cuidadores de TCE, tal como a qualidade de vida e a inclusão da pessoa com TCE ao programa reabilitador, favorecendo, assim, a continuidade da reabilitação. Ademais, o material tem como objetivo promover a inserção dos cuidadores na terapia domiciliar e favorecer a adesão da ação reabilitadora ao domicílio.

Desta forma, trata-se de um recurso auxiliador que apresenta linguagem clara e design atrativo que contribui para o ensinamento dos exercícios exercido pelo fisioterapeuta das equipes do NASF - AB e do SAD no contexto domiciliar, assim como busca proporcionar a motivação e o incentivo no aprendizado dos exercícios apresentados, por meio de instruções de fácil entendimento e frases motivadoras. Sendo assim, é uma ferramenta de saúde que busca fornecer a equidade a todo indivíduo com TCE, bem como uma melhor qualidade da ação prestada pelos serviços de reabilitação.

\section{REFERÊNCIAS}

ALMEIDA, D. Elaboração de materiais educativos. 2017. Disponível em: https://edisciplinas.usp.br/pluginfile.php/4411907/mod_resource/content/1/ELAB ORAÇÃO\%20MATERIAL\%20EDUCATIVO.pdf. Acesso em: 22 abr. 2019.

AMARAL, M. et al. Orientação domiciliar: proposta de reabilitação física para mulheres submetidas à cirurgia por câncer de mama. Revista Ciências Médicas, v. 14, n. 5, p. 405-413, 2005.

AMERICAN PHYSICAL THERAPY ASSOCIATION. Code of Ethics for the Physical Therapist. 2005. Disponível em: https://www.apta.org/uploadedFiles/APTAorg/About_Us/Policies/HOD/Ethics/Codeof Ethics.pdf. Acesso em: 19 jan. 2020. 
ANDELIC, N.; et al. Disability and quality of life 20 years after traumatic brain injury. Brain Behavior and immunity. 2018; e01018. https://doi.org/10.1002/brb3.1018.

ASSIS, L. et al. Efetividade de um manual de exercícios domiciliares na promoção da continência urinária durante a gestação: um ensaio clínico aleatorizado pragmático. Revista Brasileira de Ginecologia e Obstetrícia. v. 37, n. 10, p. 460-466, 2015.

ASSOCIAÇÃO DE MEDICINA INTENSIVA BRASILEIRA. Traumatismo Cranioencefálico (TCE) constitui a principal causa de óbitos e sequelas em pacientes multitraumatizados. 2014.

Disponível em: https://www.amib.org.br/noticia/nid/traumatismo-cranioencefalico-tce-constitui-aprincipal-causa-de-obitos-e-sequelas-em-pacientes-multitraumatizados/. Acesso: 06 out. 2019.

BORGES, C.; RODRIGUES, G. Therapeutic Exercise Protocols in Patients with Traumatic Brain Injury: A Systematic Review. Journal of Exercise Physiology Online. v. 23. n. 2, p. 71-82, 2020.

BRAGA, L.; DA PAZ, A.; YLSIVAKER, M. Direct clinican- delivered versus indirect Family- supported rehabilitation of children with traumatic brain injury: A Randomized Controlled Trial. Brain Injury. v. 19, n.10, p. 819-831, 2005.

BRASIL. Caderno de Atenção Domiciliar. Vol. 1. Brasília, 2012. p. 22.

BRASIL. Decreto no 1060, de 5 de junho de 2002. Disponível em: http://bvsms.saude.gov.br/bvs/saudelegis/gm/2002/prt1060_05_06_2002.html. Acesso em: 17 de mai. 2019

BRASIL. Ministério da Saúde. Diretrizes de Atenção à reabilitação da Pessoa com Traumatismo Cranioencefálico. Brasília, 2013. p. 12-35.

BRASIL. Núcleo Ampliado de Saúde da Família. 2018. Disponível em: https://aps.saude.gov.br/ape/nasf. Acesso em: 15 nov. 2019. 
BRASIL. Política Nacional de Saúde da Pessoa com Deficiência. Brasília, 2010. p. $1-6$.

BRASIL. Portaria n. 4.729/10. Conselho de Saúde: Redes de Atenção em Saúde (RAS). 2010. Disponível em: http://conselho.saude.gov.br/ultimas_noticias/2011/img 07_jan_portaria4279_301210. pdf. Acesso em: 15 out. 2018.

BRASIL. Práticas em Reabilitação na Atenção Básica: o olhar para a funcionalidade na interação com o território. Vol. 1. Brasília, 2017. p. 26-40.

BRASIL. Política Nacional de Saúde da Pessoa com Deficiência. 2008. Disponível em:

https://bvsms.saude.gov.br/bvs/publicacoes/politica_nacional_saude_pessoa_deficie ncia.pdf. Acesso em: 9 set. 2020.

CASTRO, S. S. et al. Acessibilidade aos Serviços de Saúde por Pessoas com Deficiência. Revista de Saúde Pública, São Paulo, v. 45, n. 1, p. 99-105, 2011.

CENTRO DE PESQUISA E ECONOMIA DO SEGURO. 2016. Estatística da dor e da perda do futuro: novas estimativas. Disponível em: https://www.ens.edu.br/arquivos/ESTATISTICAS\%20DA\%20DOR\%20E\%20DA\%20 PERDA\%20DO\%20FUTURO.pdf. Acesso em: 09 set. 2020.

ECHER, I. Elaboração de manuais de orientação para o cuidado em saúde. Revista latino-americana em enfermagem. v. 13, n. 5, p. 754-757. 2005.

FUENTES, D. et al. Neuropsicologia: teoria e prática. 2.ed. Porto Alegre: Artmed, 2014, p. 223.

FUKUJIMA, M. O traumatismo cranioencefálico na vida do brasileiro. Revista Neurociências, v. 21, n. 2, p.173-174, 2013.

GUEDES, M.; SILVA, F. Intervenção funcional e orientação aos familiares de pacientes com Acidente Vascular Cerebral (AVC) na emergência de um hospital público terciário. 2019. Disponível em: https://ceafi.edu.br/site/wp- 
content/uploads/2019/05/intervencao-funcional-e-orientacao-aos-familiares-depacientes-com-acidente-vascular-cerebral-avc-ne-emergencia-de-um-hospitalpublicotercia.pdf. Acesso em: 14 ago. 2019.

MATOS, A. et al. Reabilitação física em portadores de Legg-Calvé-Perthes após osteotomia de Salter-protocolo de orientação domiciliar. Revista ConScientae Saúde. v. 12. n. 1. p. 82-89, 2013.

MORAES, R. et al. Medicina Intensiva: Consulta Rápida. Porto Alegre: Artmed, 2014. p. $505-510$.

MOREIRA, T. et al. Tecnologia para a promoção e o cuidado em saúde. Ceará: editora UECE, 2018. p. 30.

NITRINI, R.; BACHESCHI, L. A neurologia que todo médico deve saber. 3. ed. São Paulo: Editora Atheneu, 2015. p. 187-190.

ORGANIZAÇÃO MUNDIAL DE SAÚDE. Relatório mundial sobre a deficiência. 2012. Disponível em: https://apps.who.int/iris/bitstream/handle/10665/44575/9788564047020_por.pdf?seq uence $=4$. Acesso em: 03 set. 2019 .

PETITO, E. et al. Aplicação de programa de exercícios domiciliares na reabilitação do ombro pós-cirurgia por câncer de mama. Revista Latino-Americana de Enfermagem. 2012. v. 20, n. 1, 09 telas.

POWELL, J. et al. A Telehealth Approach to Caregiver Self- Management Following Traumatic Brain Injury: A randomized Controlled Trial. Journal Head Trauma Rehabilitation. v. 31, n. 3, p. 180-189, 2016.

SILVA, R. et al. Elaboração de um manual de exercícios e orientações para pacientes com fascite plantar. Revista Acta Fisiátrica. 2014. v. 21, n. 2, p: 75-79.

SOUZA, C. C. B. X.; ROCHA, E. F. Portas de entrada ou portas fechadas?: o acesso à reabilitação nas unidades básicas de saúde da região sudeste do município de São 
Paulo - período de 2000 a 2006. Revista de Terapia Ocupacional da Universidade de São Paulo, São Paulo, v. 21, n. 3, p. 230-239, set./dez. 2010.

SOUZA, J.; KNOBEL, K. Guia ilustrado de orientações a cuidadores de crianças com deficiências neuromotoras. Revista ConScientiae Saúde. jan/mar. 2019, v. 18, n. 1, p:8-11.

SOUZA, M. et al. Fisioterapia e núcleo de apoio à saúde da família: conhecimento, ferramentas e desafios. O Mundo da Saúde, São Paulo, v.37, n. 2, p:176-18, 2013.

SOUZA, F. Rede Humaniza-SUS. Tecnologias do Cuidado em Saúde. 2013. Disponível em: http://redehumanizasus.net/65498-tecnologias-de-cuidado-emsaude/. Acesso em: 05 mai. 2020.

TORNBOM, K.; SUNNERHAGEN, K.; DANIELSSON, A. Perceptions of physical activity and walking in na early stage after stroke or acquired brain injury. PloS ONE. v. 12, n. 3, p. 1-14, 2017. doi:10.1371/journal.pone.0173463.

THINEN, N.; MORAES, A. Manual de orientação de posicionamento e execução de atividades da vida diária para pacientes com acidente vascular cerebral. Caderno de Terapia Ocupacional-UFSCar. São Carlos, 2013, v. 21, n. 1. p. 131-139.

VOIGHT, M.; HOOGENBOOM, B.; PRENTICE, W. Técnicas de exercícios terapêuticos: estratégia de intervenção musculoesquelética. 1.ed. Barueri: Manole, 2014. p. 437-438.

Enviado: Setembro, 2020.

Aprovado: Outubro, 2020. 